\title{
THE SCINTILLATION MODELS FOR SIGNAL PROPAGATION THROUGH SATTELLITE IONOSPHERIC CHANNELS
}

\section{Lev E. Nazarov, Dmitry V. Antonov, Vitaly V. Batanov, Andrey S. Zudilin, Vladimir M. Smirnov}

Kotelnikov Institute of Radioengineering and Electronics of RAS, Frayzino Branch, http:// fire.relarn.ru Fryazino 141190, Moscow Region, Russian Federation

levnaz2018@mail.ru,dmitry.vl.antonov@gmail.com, bvitaly@inbox.ru, zudilinas@hotbox.ru,vsmirnov@ sunclass.ire.rssi.ru

Abstract. The results of analysis for signals propagating through the ionosphere satellite communication channels with temporal and spatial electron density irregularities in the ionosphere plasma are presented in the article. These electron density irregularities most commonly occur in low-latitude, auroral, and polar regions and refer to the random signal amplitude and phase fluctuations. Occurrence of scintillation is difficult to predict and model due to the variability of its numerous influencing factors, which include solar activities, inter-planetary magnetic field activities, local electric field and conductivity, convection processes, wave interactions. Satellite ionospheric radio waves in P-, L- frequency bands are vulnerable to scintillations that can severely impact the acquisition and tracking process in receivers, causing a degradation in navigation and in information systems (as example, Kospas-Sarsat system) solution accuracy, integrity, and continuity. The widely-used indice to measure ionospheric scintillation activity is the scintillation index for amplitude scintillation (the standard deviation of the received signal power normalized to the average signal power). The values of scintillation index $(0.27 \ldots 0.49)$ for Cospas-Sarsat channel (406.0-406.1 MHz) are evaluated in the article. The empirical model for probability density for signal amplitude as m-law Nakagami based on these values of scintillation index is used to evaluate errorperformance degradation concerning to propagation through free space. The computer simulations for evaluation of these error-performance degradations are performed - the degradation of signal/ noise is about $6.8 \mathrm{~dB}$ for bit-error 0.001 .

Keywords: satellite ionosphere channels, signals, signal scintillations, error-performances, signals, phase shift keying signals, Kospas-Sarsat

UDC 621.391.01

Bibliography - 21 references

Received 16.04.2019, accepted 21.04.2019

RENSIT, 2019, 11(1):57-64

DOI: 10.17725/rensit.2019.11.057

\section{CONTENTS}

1. Introduction (57)

2. Problem statement (58)

3. Signal Scintillation Models (59)

4. Calculation Results (59)

5. Conclusion (62)

REFERENCES (63)

\section{INTRODUCTION}

Scintillations (fading) of signals during propagation through satellite ionospheric transmission lines are caused by random temporal and spatial fluctuations of the electron density of ionospheric inhomogeneities [1-5].

Solving problems of signal propagation through such randomly inhomogeneous media implies establishing a connection between the statistical characteristics of the electron density field and the characteristics of the signals at the input of receiving devices $[2-4,6,7]$. A fairly wide range of works $[2,4,8,9]$ is devoted to the creation and development of private models of signal scintillation. Using these models, it is possible 
to calculate the statistical characteristics of signal power variations (for example, average power, statistical power moments) and, as a result, estimate the probability characteristics when receiving signals and compare them with the probability characteristics of receiving signals when propagating in free space $[10,11]$. Based on this comparison, the corresponding energy losses are estimated, which must be taken into account in the energy budgets of radio lines.

The results of experimental and theoretical studies using these models show the dependence of energy losses due to scintillation on the central frequency, on the spatial distribution of transmitting and receiving devices, solar activity, daily time [3, 4]. Radio lines of the L-frequency range, actively used by satellite systems of global navigation (Glonass, GPS, Gallileo, etc.) [12-17], have been studied in sufficient detail. The problem of the generalization, development and addition of these results for the $\mathrm{p}$-frequency radio links, which are also actively used by satellite systems for transmitting information, is relevant. An example is the international satellite search and rescue system Cospas-Sarsat, operating in the frequency range $406.0 \ldots 406.1 \mathrm{MHz}$ [18].

\section{PROBLEM STATEMENT}

In Fig. $\mathbf{1}$ is a diagram explaining the propagation of $s(t)$ signals over a satellite ionospheric radio link. Studies show that the main contribution to the scintillation of signals is determined by the inhomogeneities of the electron density of the ionospheric layer $\mathrm{F}$, having dimensions 1 , comparable to the dimensions of the first Fresnel zone $(\lambda$ is the wavelength of the signals) $[1,4,15]$. For the location of the onboard transmitter

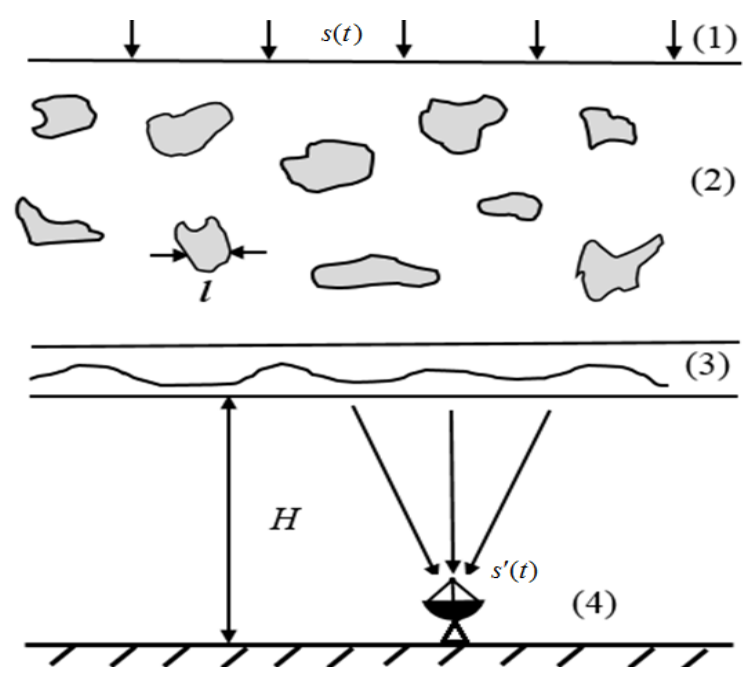

Рис. 1. Распространение синналов по спутниковой ионосферной радиолинии: 1 - плоскаяволна; 2-ионосферньиислойснеоднородностями; 3 - фазовый фронт; 4 - наземный приемный пункт.

at an altitude of $\mathrm{HO}>>\mathrm{H}(\mathrm{H}=350-400$ $\mathrm{km}$ - the height of the ionospheric layer F), which is a valid condition for satellite global navigation systems $(\mathrm{H} 0 \approx 19,200 \mathrm{~km})$, the incident signals $\mathrm{s}(\mathrm{t})$ refer to the far radiation zone and rely flat waves. In models [1, 4], the effect of ionospheric inhomogeneities is given by the action of a thin screen with a field of random phase distribution. The amplitude A of the resulting signal s' ( $t$ ) at the input of the ground receiving point is a random variable and is determined by applying diffraction methods taking into account a random field $[1,3]$.

For the basic model of channel noise in the form of additive white Gaussian noise $n$ (t) (ABGN), the rule of optimal reception of digital signals that implements the statistical maximum likelihood criterion is based on the calculation of the cross-correlation of the input implementation $s^{\prime}(t)+n(t)$ with the original signal s ( $t$ ) [10]. In this case, the probability of erroneous reception of the $\mathrm{Pb}$ bit in the coherent reception of signals with two-phase and four-phase keying 
(FM2 signals, FM4 signals) without errorcorrecting coding is determined by the relation $[10]$

$$
P_{b}\left(E_{b} / N_{0}\right)=1-F\left(\sqrt{2 E_{b} / N_{0}}\right) .
$$

Here, $\mathrm{Eb}$ is the signal energy per information bit; $\mathrm{N} 0$ is the ABGSh spectral density (one-sided);

$$
F(x)=\frac{1}{\sqrt{2 \pi}} \int_{-\infty}^{x} \exp \left(-t^{2} / 2\right) d t .
$$

Assuming the amplitude $\mathrm{A}$ of the signal $s^{\prime}(t)$ is random stationary in a broad sense of magnitude with the distribution density $\mathrm{p}$ (A), the average error probability taking into account scintillation can be calculated using the expression [10]

$$
P_{b}=\int_{0}^{\infty} P_{b}\left(E_{b} A^{2} / N_{0}\right) p(A) d A .
$$

The essence of the task is to provide descriptions of the scintillation models of the amplitudes of signals as a random process during propagation through heterogeneous satellite ionospheric radio links, to give the results of calculating the error probability for signals with phase shift keying taking into account the scintillation models and to give these signals for the P-frequency range of relation to distribution in free space.

\section{MODELS OF SIGNAL SCINTILLATION}

The considered scintillations of signals and their statistical characteristics are determined by a number of parameters - the center frequency $\mathrm{fO}$, the spatial distribution of the transmitting and receiving devices, solar activity, the speed of the ionospheric irregularities, the daily time, etc. [3, 4].

When creating and developing signals scintillation models taking into account these factors, two approaches are used - based on the use of analytical methods for describing the propagation of signals with ionospheric irregularities and on the use of empirical relationships regarding the distribution density $\mathrm{p}(\mathrm{A})[1,5]$.

In the approach based on analytical methods for describing the propagation of signals, the presence of ionospheric irregularities with small spatial variations in electron density with a scale comparable to the wavelength $\lambda[1,4]$ is considered. Its temporal variations with a 1 / f0 scale are also considered to be small. In this case, the component of the electric field $\mathrm{E}$, falling vertically on the ionospheric layer (x-axis) during propagation, is a solution of the wave equation $[3,19,20]$

$$
\frac{d^{2} E(x, t)}{d x^{2}}+k^{2} \varepsilon(x, t) E(x, t)=0 .
$$

Here $\mathrm{k}=2 \pi / \lambda$ is the wave number for free space, $\varepsilon(\mathrm{x}, \mathrm{t})$ is the dielectric constant of the medium.

Further, it is assumed that the main variations in the dielectric constant $\delta \varepsilon(\mathrm{x}, \mathrm{t}) \approx$ $4 \pi \mathrm{reNe} / \mathrm{k} 2$ occur in a limited volume with linear dimensions 1 (Fig. 1), comparable to the dimensions of the first Fresnel zone r0 (re is the electron radius). Under this condition, equation (3) is transformed into a parabolic equation $[1,3]$

$-j 2 k \frac{\partial U}{\partial x}+\nabla_{1}^{2} U+k^{2} \varepsilon(x, t) U=0$.

Here

$u=U \exp (-j k x), \quad \nabla_{1}^{2}=\frac{\partial^{2}}{\partial y^{2}}-\frac{\partial^{2}}{\partial z^{2}}$.

Equation (4) is stochastic, its solution determines the relationship between the random variables $U$ and $\varepsilon(x, t)$ and establishes their statistical characteristics (for example, statistical moments, including the fourth moment of the amplitude of the signal s' ( $t$ ), used to estimate the range of fading signals ). 
Equation (4) is stochastic, its solution determines the relationship between the random variables $\mathrm{U}$ and $\varepsilon(\mathrm{x}, \mathrm{t})$ and establishes their statistical characteristics (for example, statistical moments, including the fourth moment of the amplitude of the signal s' ( $t$ ), used to estimate the range of fading signals ).

Equation (4) is non-linear, the problem of finding its solution in the form of closed analytical expressions remains open. Its approximate solutions of Born and Rytov are known as the sum of terms depending on the small parameter $\varepsilon{ }^{\prime}(\mathrm{x}, \mathrm{t}) \quad<<1$, where $\varepsilon(\mathrm{x}, \mathrm{t})=1+\varepsilon^{\prime}(\mathrm{x}, \mathrm{t})[1,6,7]$. For the approximation $\varepsilon^{\prime}(\mathrm{x}, \mathrm{t})=0$, the solution (4) in the form of $\mathrm{U} 0(\mathrm{x}, \mathrm{t})$ determines the propagation of signals in free space. The use of the term depending on $\varepsilon$ '(x, $\mathrm{t})$ gives the Born approximation $\mathrm{U}^{\prime}(\mathrm{x}, \mathrm{t})$ (Debye-Born scattering) corresponding to single scattering under the condition $\mathrm{U} \%$ $\mathrm{U} 0<<1$ [7]. For U'/ U0 $\approx 1$, solution (4) is equivalent to solving the problem with multiple scattering [7].

The Rytov approximation (small perturbation method) is based on the use of the relation $\psi=\operatorname{Ln}(A)$, the function is a solution of the equation $[1,6,7]$.

$-2 k \frac{\partial \psi}{\partial x}+\nabla_{1}^{2} \psi+\left(\nabla_{1} \psi\right)^{2}+k^{2} \varepsilon=0$.

The solution (5) in the Rytov's approximation determines the linear relationship between the random functions and and the relationship of the statistical moments of the function $\operatorname{Ln}(\mathrm{A})$ to the amplitude of the signal $s^{\prime}(t)$ and the fluctuations of the electron density. At the same time, it is noted that the considered analytical approaches using approximate solutions of equation (4) do not provide sufficiently accurate results in the general case of the $\mathrm{U}^{\prime} / \mathrm{U} 0$ ratio with respect to experimental measurements [1].

More accurate scintillation models from the class under consideration are based on the inclusion of a thin phase screen at the height $\mathrm{H}$ of the ionosphere layer F (Fig. 1) - ionospheric irregularities disturb the screen's field, using its resultant $s^{\prime}(t)$ signals using diffraction theory methods [1, 4]. Modifications of this approach include several screens with uncorrelated phase fields [1].

Scintillation models of signals from the second class associate the parameters of empirical distribution densities $p$ (A) of the amplitude of the signal $s^{\prime}(t)$ with the scintillation index $\mathrm{S} 4=(<\mathrm{I} 2\rangle-<\mathrm{I}\rangle$ 2) $/<$ I $>2$, the values of which define an important characteristic for applications - the fading range of Pf signals [5, 9]. Here I = A2 (t) is the signal power; $<>$ Is the averaging operation over an ensemble of signals, or over time, assuming that the random process A ( $\mathrm{t}$ ) is ergodic.

The fading range of the amplitude of the Pf signals $(\mathrm{dB})$ at the input of the receiving device is determined by the approximate relationship [5]. A more accurate relationship between Pf and S4 can be determined using empirical models of the distribution density $\mathrm{p}$ (A), the parameters of which can be set using the analytical models considered above (4), (5) or using experimental studies of radio lines.

According to the values of the S4 index, fading is classified: weak to $\mathrm{S} 4<0.3$; average $0.3<\mathrm{S} 4<0.6$; strong $\mathrm{S} 4>0.6$ [5]. An increase in S4 values is accompanied by an increase in energy loss with respect to propagation in free space.

For scintillations, the density $\mathrm{p}$ (A) can be represented by well-known distribution laws: the log-normal distribution [2], the m-distribution by Nakagami [5], 
the Rayleigh-Rice distribution, the $-\alpha-\mu$ distribution [9].

Most often, the amplitude density p (A) of signals during propagation along the ionospheric radio link is described by the Nakagami distribution [4, 5]

$$
p(A)=\frac{2}{\Gamma(m)}\left(\frac{m}{\sigma^{2}}\right)^{m} A^{2 m-1} \exp \left(-\frac{m A^{2}}{\sigma^{2}}\right) .
$$

Here $\sigma 2$ is the fluctuation component of the signal power $\mathrm{s}^{\prime}(\mathrm{t}) ; \mathrm{m} \geq 1 / 2$ is a parameter specified by the relation $\Omega=<\mathrm{A} 2>$ [21]. The parameters $\mathrm{S} 4$ and $\mathrm{m}$ are related by the relation $\mathrm{m}=1 / \mathrm{S} 4$ [5].

The distribution by Nakagami is approximated by the Rayleigh-Rice distribution [21]

$$
p(A)=\frac{A}{\sigma^{2}} \exp \left(-\frac{A^{2}+A_{0}^{2}}{2 \sigma^{2}}\right) I_{0}\left(\frac{A A_{0}}{\sigma^{2}}\right) \text {. }
$$

Here, $\mathrm{A} 0$ is the average amplitude of the signal component; $\mathrm{I} 0(\mathrm{x})$ is a modified Bessel function of the first kind of zero order [21].

The Rayleigh-Rice distribution is characterized by the Rice coefficient [21]. Parameters S4, $\mathrm{m}$ and the Rice coefficient c subject to the following relations

In accordance with the experimental data for the normal mid-latitude ionosphere and for the radio link with the center frequency $\mathrm{f} 0=400 \mathrm{MHz}$, the flicker index S4 does not exceed $0.3 \ldots 0.5$, i.e. fading can be attributed to the class of weak-medium fading, for polar regions the values of S4 can reach 1 [5] and in this case fading can be attributed to the class of strong fading.

Below are the results of estimating the S4 index based on experimental measurements of the amplitudes of the signals of the Cospas-Sarsat satellite system (center frequency $\mathrm{f} 0=406 \mathrm{MHz}$ ) and the probability characteristics for receiving signals calculated using relation (2) and model descriptions of the amplitude distribution density p (A) (6), (7) with parameters corresponding to the estimates of S4.

\section{RESULTS OF CALCULATIONS}

The Cospas-Sarsat international satellite system is designed to determine the location of emergency beacons operating in the P-frequency range 406.0 ... $406.1 \mathrm{MHz}$ (uplink) and in the L-frequency range (downlink) [18]. Information messages of second-generation beacons are transmitted over a radio link using digital FM4 signals with an offset [10], a signal duration of 1 second with a nominal on-time interval of 30 seconds, and a sync sequence of $160 \mathrm{~ms}$ [18]. Relay beacon signals are located

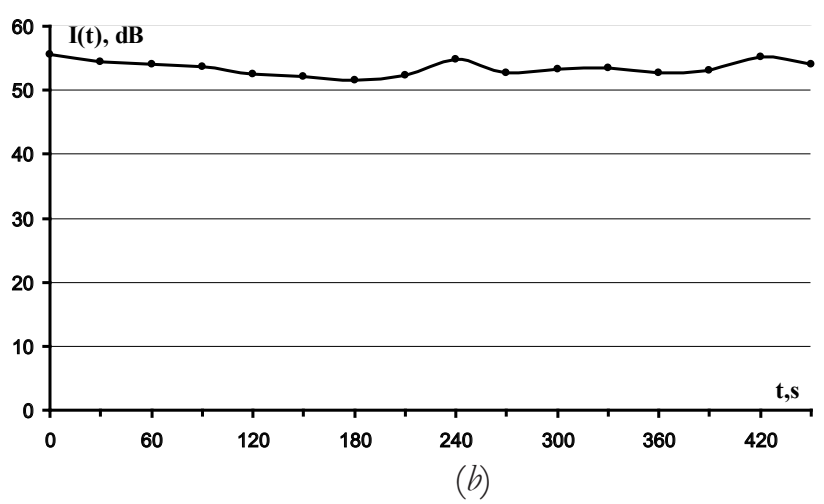

Рис. 2. Фрагменты зависимости мочности сигналов $I(t)$ от времени, полученныге путем обработки сигналов радиобуев спутниковой поиско-спасательной системьг Коспас-Сарсат второго поколения (дневное время, июль 2018 г.): (а) навигачионныгй спутник GPS; (b) навигачионный спутник Gallileo. 
on global navigation satellites (Glonass, GPS, Gallileo [17]).

In Fig. 2 shows fragments of the dependence of the power of the $I(t)$ signals on time, calculated by processing at FIRE them. V.A. Kotelnikov of the RAS of the received signals of the second generation beacons in the form of records in digital format, obtained using the technical means of the receiving station from the GPS midorbit navigation satellites (Fig. 2a), Gallileo (Fig. 2b) for daytime. Beacon producing countries - France, USA, beacons operate in test mode [18].

The peculiarity of the considered radio link is the joint influence of ionospheric irregularities on the propagation of signals in the P- (uplink) and L- (downlink) frequency ranges.

During processing, point estimates I $(t)$ were calculated for the discrete beacon turnon time by correlation processing of input realizations with a synchronization sequence.

As a result of processing signals from the Cospas-Sarsat satellite system (time period March-October 2018), a possible range of S4 scintillation index values was determined for the frequency range under study $\mathrm{S} 4=$ $0.27 \ldots 0.49$, the approximate fading range of signals amplitude Pf $=5.25 \ldots 11.20 \mathrm{db}$ The corresponding values of the Nakagami distribution parameter (6) are in the range of $\mathrm{m}=2.04 \ldots 3.70$, the corresponding range of values of the Rice coefficient of the RayleighRice distribution (7) $\mathrm{c}=4.1 \ldots 7.4$.

In Fig. 3 shows the probabilities of $\mathrm{Pb}$ error in the coherent reception of FM4 signals calculated using relations (1), (2) for the scintillation model defined by the RayleighRice amplitude distribution density (7) with the given estimates of the Rice parameter $\mathrm{c}$.

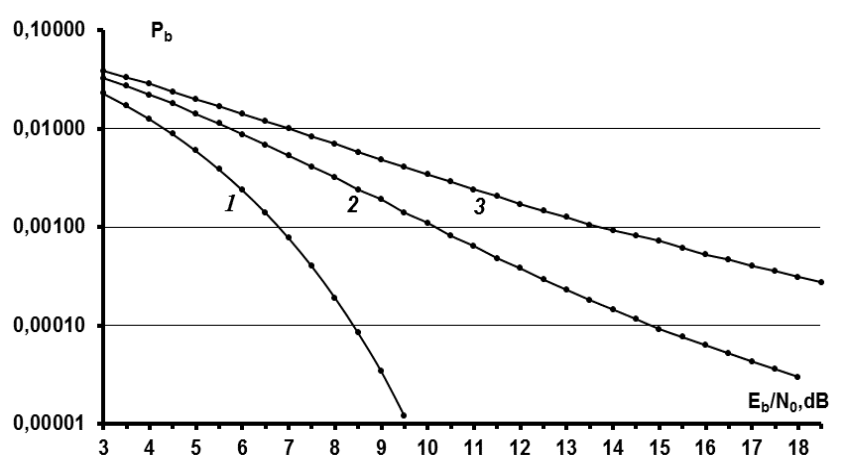

Рис. 3. Вероятности ошибки при когерентном приеме ФМ4 сигналов для модели сиинтиляячии, задаваемой плотностью распределения Релея-Райса: 1 - распространение в свободном nространстве; 2 - параметр Райса $c=7.4 ; 3$ - параметр Paŭcac $=4.1$.

Curve 1 corresponds to propagation in free space - the probability of $\mathrm{Pb}=10-3$ is ensured with respect to $\mathrm{Eb} / \mathrm{N} 0=6.7 \mathrm{~dB}$.

Curve 2 corresponds to the maximum value of the Rice parameter in the range of their estimates $\mathrm{c}=7.4$, the probability $\mathrm{Pb}=$ $10-3$ is ensured at the ratio $\mathrm{Eb} / \mathrm{N} 0=10.0$ $\mathrm{dB}$, the energy loss relative to curve 1 reaches $3.3 \mathrm{~dB}$.

Curve 3 corresponds to the minimum value of the Rice parameter in the range of their estimates $c=4.1$. It can be seen that the probability of $10-3$ is ensured with respect to $\mathrm{Eb} / \mathrm{N} 0=13.5 \mathrm{~dB}$, which corresponds to the energy loss with respect to curve 1 to $6.8 \mathrm{~dB}$.

Energy losses increase with decreasing $\mathrm{Pb}$ error values.

\section{CONCLUSION}

The descriptions of the models of scintillation (fading) of signals due to random temporal and spatial fluctuations of the electron density of ionospheric inhomogeneities are given. These models fall into two general classes - based on the application of analytical methods for describing signal propagation using the theory of stochastic equations and on the basis of empirical models regarding 
the density distribution of signal amplitudes at the input of receivers using a scintillation index (fourth-order statistical moment of signal amplitudes).

The results of the numerical estimation of the scintillation index for the mid-latitude radio line of the Cospas-Sarsat satellite information system are given. A feature of this radio link is the joint influence of ionospheric irregularities on the propagation of signals in the P- (uplink) and L- (downlink) frequency ranges. As a result of signal processing of the Cospas-Sarsat system, a possible range of scintillation index values of $0.27 \ldots 0.49$ was determined. Using the empirical scintillation model, we calculated the probability characteristics of receiving signals with phase shift keying distributed over the radio link under consideration with a given scintillation index range, and determined the required power margin of up to $6.8 \mathrm{~dB}$ for the error probability 0.001 with respect to spreading in free space. This must be taken into account when calculating the energy budget of a given radio link.

The study of the statistical characteristics of the studied radio link, in particular, the determination of the time and frequency band of coherence, as well as the specification of the range of the energy reserve, taking into account the noise-resistant coding methods, constitute the direction of prospective studies.

\section{REFERENCES}

1. Crane RK. Ionospheric Scintillation. Proceeding of IEEE, 1977, 2: 180-199.

2. Kolosov MA, Armand NA, Yakovlev OI. The propagation of radio waves in space communications. M., Communication, 1969, 156 p.
3. Yakovlev OI, Yakubov VP, Uryadov VP, Pavelev AG. The propagation of radio waves. M., LENAND, 2009, 496 c.

4. Rino CL. Remote Sensing. John Wiley \& Sons, Hoboken, New Jersey, 2011, 244 p.

5. Satellite services and systems. Recommendation ITU-R P.531-11. Electronic Publication, Geneva, 2012, 24 p.

6. Rytov SM, Kravtsov YuA, Tatar VI. Introduction to statistical radiophysics. T. 2. Random fields. M., Science, 1978, 464 c.

7. Isimaru A. Propagation and scattering of waves in random environments. T. 2. M., Mir, 1981, 280 p.

8. Priyadarshi S. Review of Ionospheric Scintillation Models. Surveys in Geophysics, 2015. 36 (2): 295-324.

9. Moraes AO, Vani BC, CostaE, Sousasantos J, Abdu MA, Rodrigues F, Gladek YC, Oliveira BA, Monico JF. Ionospheric Scintillation Fading Coefficients for GPS L1, L2, and L5 Frequencies. Radio Science, 2018, 53: 1165-1174.

10. Sklyar B. Digital communication. Theoretical foundations and practical application. M., Ed. Williams House, 2003, 1104 p.

11. Nazarov LE, Batanov VV. Probabilistic characteristics of the detection of radio pulses during the propagation of satellite communication systems over the ionospheric transmission lines. Technology and Electronics, 2017, 62 (9): 866-874.

12. Humphreys TE, Psiaki ML, Kintner PM. Modeling the effects of ionospheric scintillation on GPS carrier phase tracking. IEEE Transactions on Aerospace and Electronic Systems, 2010, 46 (4): 1624-1637. 
13. Ghafoori F, Skone S. Impact of equatorial ionospheric irregularities on GNSS receivers using real and synthetic scintillation signals. Radio Science, 2015, 50: 294-317.

14.Jiao Y, Morton Y, Taylor S, Pelgrum W. Characterization of high-latitude ionospheric scintillation of GPS signals. Radio Science, 2013, 48 (6): 698-708.

15. Jiao $\mathrm{Y}, \mathrm{Xu} \mathrm{D}$, Morton $\mathrm{Y}$, Rino CL. Equatorial scintillation amplitude fading characteristics across the GPS frequency bands. Navigation, 2016, 63 (3): 267-281.

16. Hofinann-Wellenhof B, Lichtenegger $\mathrm{H}$, Collins J. Global Positioning System. Theory and Practice. Springer-Verlag, New York, 1994, 392 p.

17. GLONASS: principles of construction, functioning. M .: Radio engineering. 2010, $798 \mathrm{~s}$.

18. Specification for second-generation COSPAS-SARSAT $406 \mathrm{MHz}$ distress beacons. C / S T.018. Issue 1, 2016.

19. Kutuza BG, Moshkov AV, Pozhidaev VN. Combined method that eliminates the influence of the ionosphere when processing signals of P-range airborne radars with synthetic aperture. Radio engineering and electronics, 2015, 60 (9): 889-895.

20. Bova YI, Kryukovsky AS, Lukin DS. Propagation of frequency-modulated radiation of electromagnetic waves in the Earth's ionosphere, taking into account absorption and an external magnetic field. Radio engineering and electronics, 2019, 64 (1): 3-14.

21. Tikhonov VI. Statistical radio engineering. Moscow, Soviet Radio, 1966, 678 p. 